\title{
Application of I8F-FDG PET/CT combined with carbohydrate antigen 19-9 for differentiating pancreatic carcinoma from chronic mass-forming pancreatitis in Chinese elderly
}

This article was published in the following Dove Press journal:

Clinical Interventions in Aging

29 September 2016

Number of times this article has been viewed

Xinjin $\mathrm{Gu}^{\prime}$

Rong Liu ${ }^{2}$

'Department of Hepatobiliary Surgery, Chinese People's Liberation Army General Hospital and Hainan Branch, Sanya, ${ }^{2}$ Department of Hepatobiliary and Pancreatic Surgical Oncology, Chinese People's Liberation Army General Hospital, Beijing, People's Republic of China
Correspondence: Rong Liu

Department of Hepatobiliary and Pancreatic Surgical Oncology, Chinese People's Liberation Army General Hospital, Fuxing Road 28, Beijing 100853 , People's Republic of China Email liurong30I@I26.com
Objective: The current study was designed to analyze the value of $18 \mathrm{~F}-\mathrm{FDG}$ positron emission tomography/computed tomography (PET/CT) combined with carbohydrate antigen 19-9 (CA19-9) in differentiating pancreatic carcinoma (PC) from chronic mass-forming pancreatitis (CMFP) in Chinese elderly.

Methods: As it is impossible to differentially diagnose PC from CMFP, 60 participants older than 65 years with focal pancreatic lesions were scanned by 18F-FDG PET/CT and their CA19-9 levels were tested. Diagnoses of all participants were confirmed by comprehensive methods including aspiration biopsy, surgical pathology, and clinical follow-up of 12 months. Twenty participants with CMFP were included in CMFP group and 40 participants with PC in PC group. Results: In CMFP and PC groups, 46 participants showed increased 18F-FDG uptake, 43 had elevated CA19-9 levels, and 38 participants had both increased 18F-FDG uptake and elevated CA19-9 levels. Standardized uptake value maximum of PC group (5.98 \pm 2.27$)$ was significantly different from CMFP group $(2.58 \pm 1.81, P<0.05)$. Sensitivity, specificity, and accuracy of 18F-FDG PET/CT in differentiating PC from CMFP were $95 \%, 60 \%$, and $83.3 \%$, respectively. CA19-9 levels of PC group $(917.44 \pm 1,088.24)$ were significantly different from CMFP group $(19.09 \pm 19.54, P<0.05)$. Sensitivity, specificity, and accuracy of CA19-9 levels in differentiating PC from CMFP were $87.5 \%, 60 \%$, and $78.3 \%$, respectively. Sensitivity, specificity, and accuracy of 18F-FDG PET/CT combined with CA19-9 levels in differentiating PC from CMFP were $90 \%, 90 \%$, and $90 \%$, respectively.

Conclusion: 18F-FDG PET/CT had reliable sensitivity, specificity, and accuracy in differentiating PC from CMFP, and CA19-9 levels could be helpful in 18F-FDG PET/CT for differentiating PC from CMFP in Chinese elderly. Moreover, 18F-FDG PET/CT combined with CA19-9 levels was found to be an effective method to differentially diagnose PC from CMFP and has paved the way for the timely and safe treatment of PC and CMFP in Chinese elderly.

Keywords: carbohydrate antigen 19-9, Chinese elderly, chronic mass-forming pancreatitis, 18F-FDG PET/CT, pancreatic carcinoma

\section{Introduction}

Pancreatic carcinoma (PC) has become a common malignant tumor accounting for $8 \%-10 \%$ of digestive system malignancy. ${ }^{1}$ It is difficult to be diagnosed and cured, and it spreads and relapses easily; it is the fourth leading cause of cancer-related death. ${ }^{2}$ Chronic mass-forming pancreatitis (CMFP) is a special type of chronic pancreatitis associated with autoimmune reaction, diseases due to drinking, and biliary diseases, 
and accounts for $10 \%-30 \%$ of chronic pancreatitis. ${ }^{3}$ Both PC and CMFP are focal pancreatic lesions and show very similar symptoms, signs, and imaging performance. Differential diagnosis between PC and CMFP is very difficult, and misdiagnosis and mistreatment of PC and CMFP have serious impacts on patients. ${ }^{4}$ Noninvasive imaging examinations including abdominal ultrasound, computed tomography, and magnetic resonance imaging provide insufficient information regarding differential diagnosis between PC and CMFP. ${ }^{5}$ As one kind of imaging method combining anatomical image with functional metabolism, 18F-FDG positron emission tomography/ computed tomography (PET/CT) has the potential to make a differential diagnosis of focal pancreatic lesions. ${ }^{6,7}$ However, the studies exploring the application of $18 \mathrm{~F}-\mathrm{FDG}$ PET/CT for differential diagnosis of focal pancreatic lesions are limited, especially in Chinese elderly. ${ }^{8}$ Carbohydrate antigen 19-9 (CA19-9) is a cancer marker often used to screen PC, but CA19-9 levels are also found to be abnormal in patients with CMFP as well as in those with numerous benign and malignant diseases of hepatobiliary and gastrointestinal systems and, therefore, CA19-9 cannot be used solely in differentially diagnosing PC from CMFP. ${ }^{9}$ The current study was designed to analyze the value of 18F-FDG PET/CT combined with CA19-9 in differentiating PC from CMFP in Chinese elderly.

\section{Methods}

\section{Study participants}

As it is impossible to differentially diagnose PC from CMFP, 60 participants older than 65 years with focal pancreatic lesions were scanned by 18F-FDG PET/CT and their CA19-9 levels were tested. They showed similar symptoms, signs, and imaging performance including abdominal ultrasound, computed tomography, and magnetic resonance imaging. Diagnoses of all participants were confirmed by comprehensive methods including aspiration biopsy, surgical pathology, and clinical follow-up of 12 months. Twenty participants with CMFP were included in CMFP group and 40 participants with PC in PC group. This study was approved by the Ethics Committee of Chinese People's Liberation Army General Hospital and performed according to the Declaration of Helsinki. Each participant provided written informed consent to be included in the study and their images to be published in this paper.

\section{Image interpretation and blood test}

All participants were injected with $18 \mathrm{~F}-\mathrm{FDG}(5.55 \mathrm{MBq} / \mathrm{kg})$ and scanned by Siemens Biograph 64 high-definition PET/CT. Scanned area ranged from the skull base to the upper femur, and obtained image was reconstructed by ordered subset expectation maximization. Standardized uptake value maximum (SUVmax) levels were measured from region of interest in 18F-FDG PET/CT image through semi-quantity analysis. Blood samples were drawn from all participants by venipuncture and CA19-9 levels were tested by automated electrochemical luminescence immunoassay. SUVmax levels more than 3.0 were referred to as increased 18F-FDG uptake and CA19-9 levels more than $37 \mathrm{U} / \mathrm{mL}$ as elevated CA19-9 levels. ${ }^{10}$

\section{Statistical analyses}

Continuous variables were expressed as mean (standard deviation) or median (interquartile range) and analyzed by Student's $t$ test or Mann-Whitney $U$ test. Categorical variables were expressed as number (percentage) and analyzed by chi-square test. Bivariate correlations were assessed by Pearson or Spearman coefficients. All analyses were performed by Statistical Package for Social Sciences (SPSS) version 17.0 (SPSS Inc, Chicago, IL, USA) software, and a two-tailed test with $P<0.05$ was considered to be statistically significant.

\section{Results}

Of the study participants, 38 were men $(63.3 \%)$ with a mean age of $69 \pm 7.1$ years. Table 1 shows the patients characteristics. In CMFP and PC groups, 46 participants showed increased 18F-FDG uptake. Among 46 participants, 38 had PC and eight had CMFP. Among the remaining 14 participants with normal 18F-FDG uptake, two had PC and 12 had CMFP. Sensitivity, specificity, and accuracy of 18F-FDG PET/CT in differentiating PC from CMFP were $95 \%, 60 \%$, and $83.3 \%$, respectively (Table 2). SUVmax of PC group (5.98 \pm 2.27$)$ was significantly different from CMFP group $(2.58 \pm 1.81, P<0.05)$. Figures 1 and 2 are the images obtained from two participants with CMFP and PC, respectively.

In CMFP and PC groups, 43 participants showed elevated CA19-9 levels, among whom, 35 had PC and eight

Table I Characteristics of study participants

\begin{tabular}{ll}
\hline Characteristics & Study participants \\
\hline Age, mean \pm SD & $69 \pm 7.1$ \\
Sex, $n(\%)$ & $38(63.3 \%)$ \\
BMI, mean \pm SD & $24 \pm 5.8$ \\
PC, $n(\%)$ & $40(66.7 \%)$ \\
CMFP, $n(\%)$ & $20(33.3 \%)$ \\
Diabetes mellitus, n (\%) & $9(15 \%)$ \\
\hline
\end{tabular}

Abbreviations: BMI, body mass index; PC, pancreatic carcinoma; CMFP, chronic mass-forming pancreatitis; SD, standard deviation. 
Table 2 Application of I8F-FDG PET/CT and CAI9-9 in differentially diagnosing PC from CMFP

\begin{tabular}{|c|c|c|c|c|c|c|}
\hline Characteristics & Status & PC, $n$ & CMFP, n & Sensitivity & Specificity & Accuracy \\
\hline \multirow[t]{2}{*}{ I8F-FDG uptake } & Increased & 38 & 8 & $95 \%$ & $60 \%$ & $83.3 \%$ \\
\hline & Normal & 2 & 12 & & & \\
\hline \multirow[t]{2}{*}{ CA19-9 levels } & Elevated & 35 & 8 & $87.5 \%$ & $60 \%$ & $78.3 \%$ \\
\hline & Normal & 5 & 12 & & & \\
\hline I8F-FDG uptake & Both & 36 & 2 & $90 \%$ & $90 \%$ & $90 \%$ \\
\hline and CAI9-9 levels & Other & 4 & 18 & & & \\
\hline
\end{tabular}

Abbreviations: CA19-9, carbohydrate antigen 19-9; PC, pancreatic carcinoma; CMFP, chronic mass-forming pancreatitis.

had CMFP. Among the remaining 17 participants with normal CA19-9 levels, five had PC and 12 had CMFP. Sensitivity, specificity, and accuracy of CA19-9 levels in differentiating PC from CMFP were $87.5 \%, 60 \%$, and $78.3 \%$, respectively (Table 2). CA19-9 levels of PC group (917.44 $\pm 1,088.24)$ were significantly different from CMFP group (19.09 \pm 19.54 , $P<0.05)$. There were significant correlations between
SUVmax and CA19-9 levels in CMFP $(r=0.881, P<0.05)$ and PC $(r=0.439, P<0.05)$ groups. Scatter plots for SUVmax and CA19-9 levels in CMFP and PC groups are shown in Figures 3 and 4.

Of the study participants in both CMFP and PC groups, 38 participants showed both increased 18F-FDG uptake and elevated CA19-9 levels. Among the 38 participants,
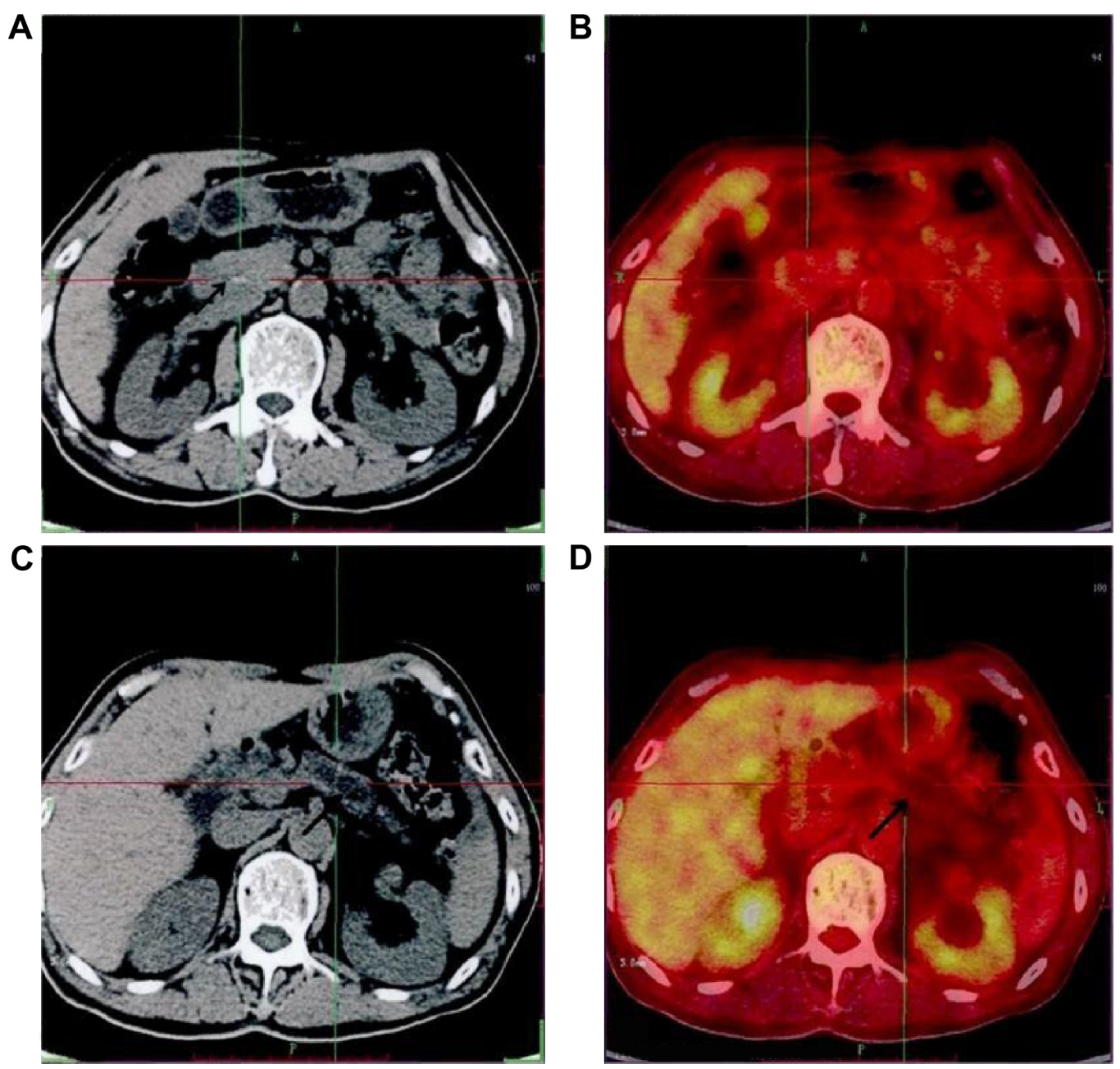

Figure I Images of a male participant, 69 years.

Notes: Computed tomography showed that there was a suspicious lesion in his pancreas. His carbohydrate antigen 19-9 level was 5I.68. I8F-FDG PET/CT showed that there was no lesion with increased I8F-FDG uptake in his pancreas. Finally, he was diagnosed with chronic mass-forming pancreatitis. (A) Pancreatic head lesion in computed tomography; (B) pancreatic head lesion in I8F-FDG PET/CT; (C) pancreatic duct dilatation in computed tomography; and (D) pancreatic duct dilatation in I8F-FDG PET/CT. Abbreviations: PET, positron emission tomography; CT, computed tomography. 

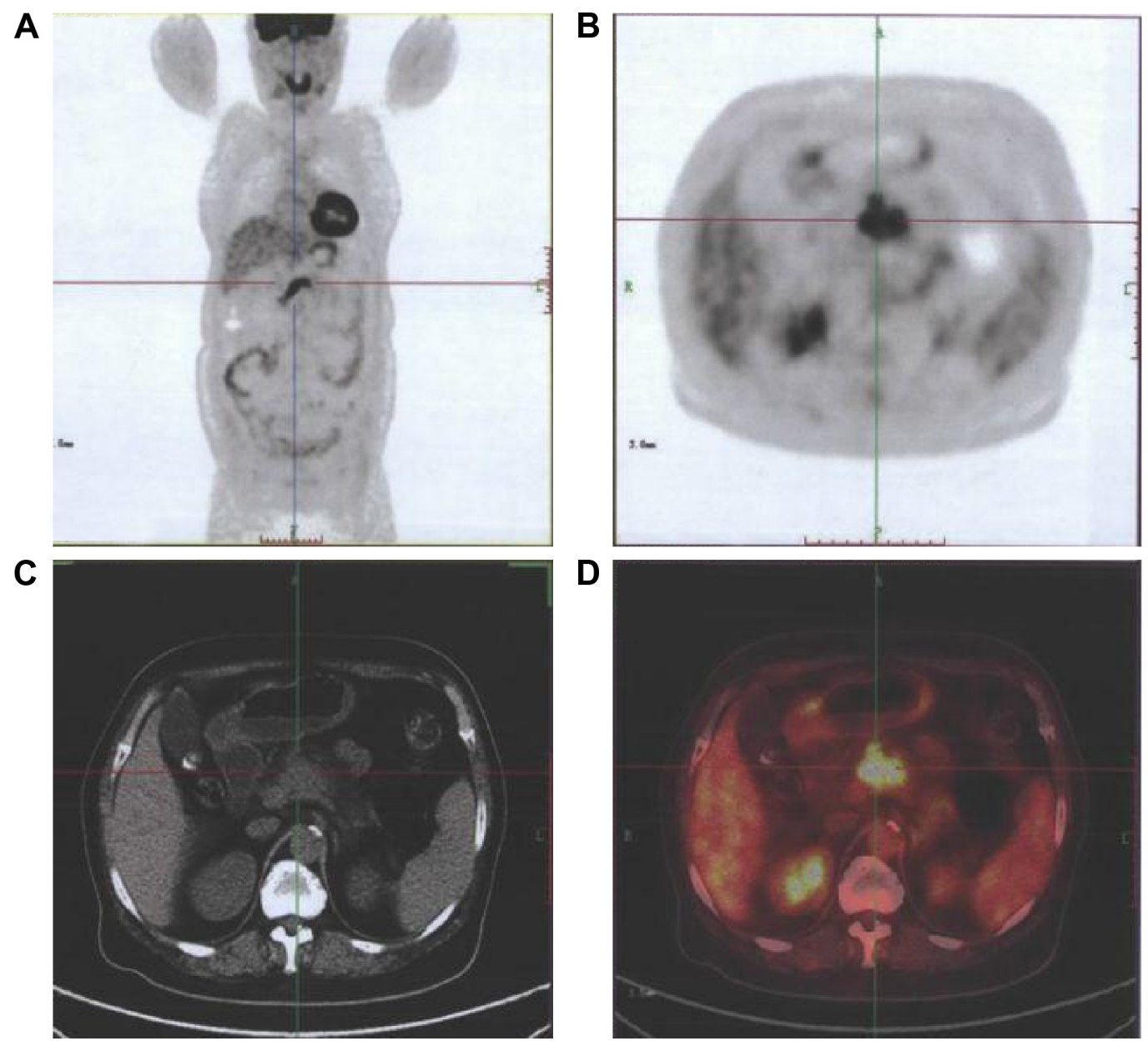

Figure 2 Images of a female participant, 66 years.

Notes: Computed tomography showed that there was a suspicious lesion in her pancreas. Her carbohydrate antigen I9-9 level was I0I5. I8F-FDG PET/CT showed that there was a lesion with increased I8F-FDG uptake (standardized uptake value maximum $=6.34$ ) in her pancreas. Finally, she was diagnosed with pancreatic carcinoma. (A) Coronal view of images obtained by I8F-FDG PET; (B) horizontal view of images obtained by I8F-FDG PET; (C) horizontal view of images obtained by CT; (D) and horizontal view of images obtained by I8F-FDG PET/CT.

Abbreviations: PET, positron emission tomography; CT, computed tomography.

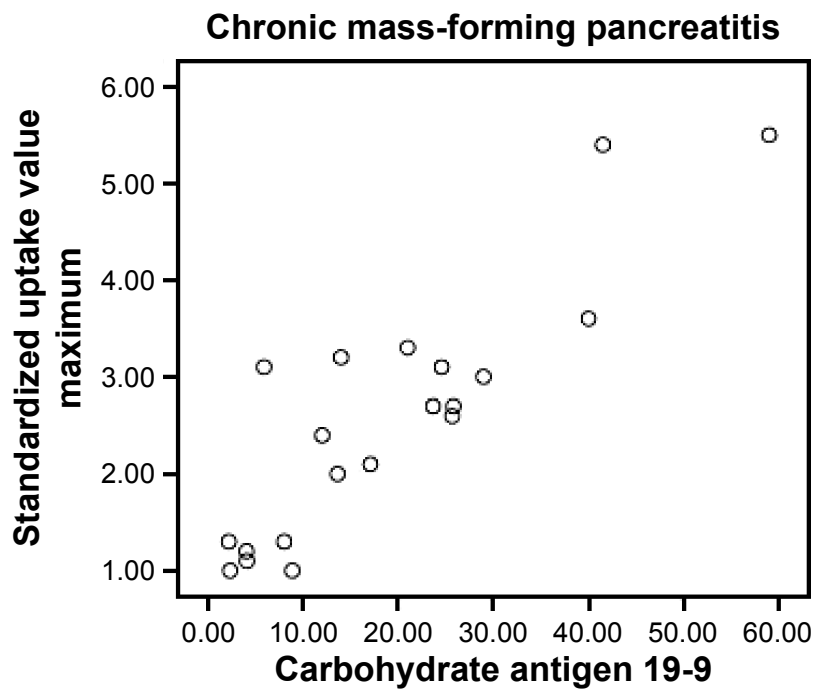

Figure 3 Scatter plot for participants with chronic mass-forming pancreatitis between standardized uptake value maximum and carbohydrate antigen 19-9 levels.

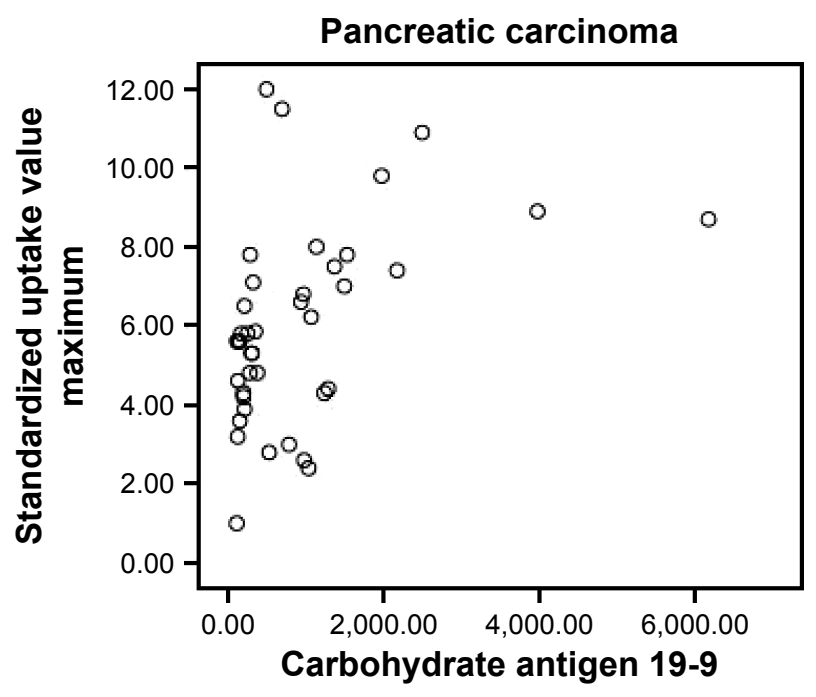

Figure 4 Scatter plot for participants with pancreatic carcinoma between standardized uptake value maximum and carbohydrate antigen 19-9 levels. 
36 had PC and two had CMFP. Among the remaining 22 participants, four had PC and 18 had CMFP. Sensitivity, specificity, and accuracy of 18F-FDG PET/CT combined with CA19-9 levels in differentiating PC from CMFP were $90 \%, 90 \%$, and 90\%, respectively (Table 2).

\section{Discussion}

PC has an increasing trend of prevalence and accounts for $1 \%-2 \%$ of malignant tumor. ${ }^{1}$ It is difficult to be diagnosed and cured, and spreads and relapses easily; it is the fourth leading cause of cancer-related death. ${ }^{2}$ CMFP, also known as inflammatory pancreatic mass, accounts for $10 \%-30 \%$ of chronic pancreatitis. ${ }^{3}$ PC and CMFP can be easily misdiagnosed which has a negative impact on patients, family, and society; therefore, it is high time that an accurate differential diagnosis between PC and CMFP is made possible. ${ }^{4}$ Symptoms and signs of PC and CMFP are very similar and, therefore, cannot be relied on for differential diagnosis. Because both PC and CMFP are focal pancreatic lesions, noninvasive imaging examinations including abdominal ultrasound, computed tomography, and magnetic resonance imaging have a limited effect on differential diagnosis between PC and CMFP. ${ }^{5}$ Use of invasive means such as surgical exploration and aspiration biopsy will not only lead to misdiagnosis and mistreatment due to deviations of draw materials but also to risk of bleeding and pancreatic fistula.

$18 \mathrm{~F}-\mathrm{FDG}$ PET/CT is a high-tech imaging equipment that not only accurately indicates anatomical image but also effectively displays functional metabolism. ${ }^{11,12}$ As PC cells grow rapidly and anaerobic glycolysis increases, 18F-FDG was taken up more by $\mathrm{PC}$ cells, which are converted into 6-P-18F-FDG and stored in PC cells. 18F-FDG PET can clearly show PC focus with metabolic boost and 18F-FDG concentration and have the ability to make a differential diagnosis of focal pancreatic lesions. ${ }^{6,7}$ Reske et $\mathrm{al}^{13}$ found that SUVmax of PC was obviously higher than that of CMFP. Prior studies have explored the application of $18 \mathrm{~F}-\mathrm{FDG}$ PET/CT for the diagnosis of PC, but these studies were limited, especially in Chinese elderly. ${ }^{8}$ The current study found that 18 F-FDG PET/CT had reliable sensitivity, specificity, and accuracy in differentiating PC from CMFP in Chinese elderly.

As a cancer marker often applied to screen the PC, CA19-9 is a mucoprotein present in pancreatic, biliary, gastric, and intestinal epithelium cells. ${ }^{14}$ On the one hand, PC cells grow up, invade, and injure the normal pancreatic and biliary cells leading to the release of CA19-9; on the other hand, PC cells themselves release CA19-9 as they grow up. False-negative errors are obtained in the following conditions.
When PC is small without or with light obstructive jaundice, CA19-9 levels are normal. When PC is big, CA19-9 levels are normal for $10 \%$ of people with Lewis-a negative because of their inability to release CA19-9. ${ }^{15}$ Not only the condition Lewis-a negative but also diabetes affects CA19-9 levels. False-positive errors are observed in patients not only with CMFP but also with numerous benign and malignant diseases of hepatobiliary and gastrointestinal systems. ${ }^{16}$ For these reasons, CA19-9 levels are not solely used for the differential diagnosis of PC from CMFP. ${ }^{9}$ The current study suggested that CA19-9 levels could be helpful in 18F-FDG PET/CT for differentiating PC from CMFP in Chinese elderly.

\section{Conclusion}

The current study confirmed that $18 \mathrm{~F}-\mathrm{FDG}$ PET/CT combined with CA19-9 levels can be used as an effective method to make a differential diagnosis between PC and CMFP, and has paved the way for the timely and safe treatment of PC and CMFP in Chinese elderly.

\section{Disclosure}

The authors report no conflicts of interest in this work.

\section{References}

1. Maisonneuve P, Lowenfels AB. Epidemiology of pancreatic cancer: an update. Dig Dis. 2010;28(4-5):645-656.

2. Siegel R, Naishadham D, Jemal A. Cancer statistics, 2012. CA Cancer J Clin. 2012;62(1):10-29.

3. Spanier BW, Dijkgraaf MG, Bruno MJ. Epidemiology, aetiology and outcome of acute and chronic pancreatitis: an update. Best Prac Res Clin Gastroenterol. 2008;22(1):45-63.

4. Tajima Y, Kuroki T, Tsutsumi R, Isomoto I, Uetani M, Kanematsu T. Pancreatic carcinoma coexisting with chronic pancreatitis versus tumorforming pancreatitis: diagnostic utility of the time-signal intensity curve from dynamic contrast-enhanced MR imaging. World J Gastroenterol. 2007;13(6):858-865.

5. Martina MC, Cassinis MC, Tappero C, Gandini G. Non invasive methods in competition for EUS (US, CE-US, CT, MRI and PET/CT): the radiologist point of view. Minerva Med. 2007;98(4):343-350.

6. Kauhanen SP, Komar G, Seppänen MP, et al. A prospective diagnostic accuracy study of $18 \mathrm{~F}$-fluorodeoxyglucose positron emission tomography/computed tomography, multidetector row computed tomography, and magnetic resonance imaging in primary diagnosis and staging of pancreatic cancer. Ann Surg. 2009;250(6):957-963.

7. Farma JM, Santillan AA, Melis M, et al. PET/CT fusion scan enhances CT staging in patients with pancreatic neoplasms. Ann Surg Oncol. 2008; 15(9):2465-2471

8. Zimny M, Fass J, Bares R, et al. Fluorodeoxyglucose positron emission tomography and the prognosis of pancreatic carcinoma. Scand $J$ Gastroenterol. 2000;35(8):883-888.

9. Dranka-Bojarowska D, Lekstan A, Olakowski M, et al. The assessment of serum concentration of adiponectin, leptin and serum carbohydrate antigen-19.9 in patients with pancreatic cancer and chronic pancreatitis. J Physiol Pharmacol. 2015;66(5):653-663.

10. Friess H, Langhans J, Ebert M, et al. Diagnosis of pancreatic cancer by $2[18 \mathrm{~F}]$-fluoro-2-deoxy-D-glucose positron emission tomography. Gut. 1995;36(5):771-777. 
11. Ell PJ. PET/CT in oncology: a major technology for cancer. Chang Gung Med J. 2005;28(5):274-283.

12. Delbeke D, Pinson CW. Pancreatic tumors: role of imaging in the diagnosis, staging and treatment. $J$ Hepatobiliary Pancreat Surg. 2004;11(1):4-10.

13. Reske SN, Grillenberer KG, Glatting G, et al. Overexpression of glucose transporter 1 and increased FDG uptake in pancreatic carcinoma. J Nucl Med. 1997;38(9):1344-1348.

14. Ballehaninna UK, Chamberlain RS. The clinical utility of serum CA 19-9 in the diagnosis, prognosis and management of pancreatic adenocarcinoma: an evidence based appraisal. J Gastrointest Oncol. 2012; 3(2):105-119
15. Okusaka T, Yamada T, Maekawa M. Serum tumor markers for pancreatic cancer: the dawn of new era? J Pancreas. 2006;7(4):332-336.

16. Singh S, Tang SJ, Sreenarasimhaiah J, Lara LF, Siddiqui A. The clinical utility and limitations of serum carbohydrate antigen (CA 19-9) as a diagnostic tool for pancreatic cancer and cholangiocarcinoma. Dig Dis Sci. 2011;56(8):2491-2496.
Clinical Interventions in Aging

\section{Publish your work in this journal}

Clinical Interventions in Aging is an international, peer-reviewed journal focusing on evidence-based reports on the value or lack thereof of treatments intended to prevent or delay the onset of maladaptive correlates of aging in human beings. This journal is indexed on PubMed Central, MedLine,

\section{Dovepress}

CAS, Scopus and the Elsevier Bibliographic databases. The manuscript management system is completely online and includes a very quick and fair peer-review system, which is all easy to use. Visit http://www.dovepress. com/testimonials.php to read real quotes from published authors. 